\title{
ON THE UNIVERSALITY OF THE GLOBAL DENSITY SLOPE-ANISOTROPY INEQUALITY
}

\author{
Emmanuel Van Hese, Maarten Baes, and Herwig Dejonghe \\ Sterrenkundig Observatorium, Universiteit Gent, Krijgslaan 281 S9, B-9000 Gent, Belgium; emmanuel.vanhese@ gmail.com, \\ maarten.baes@ugent.be, herwig.dejonghe@ugent.be \\ Received 2010 October 4; accepted 2010 October 20; published 2010 December 17
}

\begin{abstract}
Recently, some intriguing results have led to speculations whether the central density slope-velocity dispersion anisotropy inequality (An \& Evans) actually holds at all radii for spherical dynamical systems. We extend these studies by providing a complete analysis of the global slope-anisotropy inequality for all spherical systems in which the augmented density is a separable function of radius and potential. We prove that these systems indeed satisfy the global inequality if their central anisotropy is $\beta_{0} \leqslant 1 / 2$. Furthermore, we present several systems with $\beta_{0}>1 / 2$ for which the inequality does not hold, thus demonstrating that the global density slope-anisotropy inequality is not a universal property. This analysis is a significant step toward an understanding of the relation for general spherical systems.
\end{abstract}

Key words: dark matter - Galaxy: kinematics and dynamics - methods: analytical

\section{INTRODUCTION}

Theoretical dynamical models continue to play a key role in stellar dynamics, as understanding their underlying structure helps shed light on the properties of numerical and observational stellar systems and dark matter halos. In this paper, we focus our attention on the relation between the density slope $\gamma(r)$ and the velocity anisotropy profile $\beta(r)$, which has attracted renewed interest lately. As is well known, An \& Evans (2006) proved that the central inequality $\gamma_{0} \geqslant 2 \beta_{0}$ is a necessary condition for the positivity of the distribution function (DF) of a spherical system. More recently however Ciotti \& Morganti (2010a, $2010 \mathrm{~b}$ ) showed that $\gamma(r) \geqslant 2 \beta(r)$ at all radii (hereafter called the global density slope-anisotropy relation, GDSAI) is a necessary condition for positivity of the DF, if $\beta_{0} \leqslant 1 / 2$, in the families of multi-component Osipkov-Merritt (Osipkov 1979; Merritt 1985), Cuddeford (Cuddeford 1991), and Cuddeford-Louis models (Cuddeford \& Louis 1995), as well as for the Plummer models of Dejonghe (1987), the Hernquist models of Baes \& Dejonghe (2002), and the models we introduced in Baes \& van Hese (2007, hereafter Paper I). Their proof is based on the fact that all these models are characterized by having a separable augmented density (see Section 3). They also note that currently no counterexamples of the GDSAI are known, but remark that in the case of Cuddeford models with a central anisotropy $\beta_{0}>1 / 2$ the GDSAI is only a sufficient condition, so that possible counterexamples could be found in this range of values.

These results pose the question under which conditions the GDSAI holds for all spherical systems. In this paper, we make important advancements by providing a complete analysis of the GDSAI for all well-behaved systems with a separable augmented density. This group includes all aforementioned models, as well as the hypervirial models of Evans \& An (2005), the $\gamma$-models of Buyle et al. (2007), and the Dehnen-McLaughlin systems discussed in Van Hese et al. (2009, hereafter Paper II), among others. First, we show that the GDSAI holds for all separable systems, if $\beta_{0} \leqslant 1 / 2$, by proving an equivalent criterion formulated by Ciotti \& Morganti (2010b). In this manner, we extend their previous results. Our analysis also reveals some very peculiar properties of separable systems. Furthermore, we show that counterexamples of the GDSAI do exist for separable systems with $\beta_{0}>1 / 2$; in other words, we demonstrate that the GDSAI is not a universal property. However, the velocity distributions of these models are extreme, and all counterexamples are very likely dynamically unstable.

First, we outline in Section 2 the general concepts of spherical dynamical models. In Section 3, we describe the augmented density framework. In Section 4, we give our analysis of the GDSAI for separable systems: we prove the inequality for models with $\beta_{0} \leqslant 1 / 2$, and we present three counterexamples with $\beta_{0}>1 / 2$. Finally, we discuss our results in Section 5 .

\section{SPHERICAL DYNAMICAL MODELS}

The dynamical structure of a spherical gravitational equilibrium system, governed by a positive potential $\psi(r)$, is completely determined by the non-negative phase-space $\operatorname{DF} F(\vec{r}, \vec{v})$. For spherical systems, this DF is a function $F(E, L)$ of the isolating integrals, the binding energy $E$, and the angular momentum $L$ :

$$
\begin{gathered}
E=\psi(r)-\frac{1}{2} v_{r}^{2}-\frac{1}{2} v_{T}^{2}, \\
L=r v_{T},
\end{gathered}
$$

with

$$
v_{T}=\sqrt{v_{\theta}^{2}+v_{\varphi}^{2}},
$$

the transverse velocity. From the DF, the velocity moments

$$
\mu_{2 n, 2 m}(r)=2 \pi M \iint F(E, L) v_{r}^{2 n} v_{T}^{2 m+1} d v_{r} d v_{T}
$$

can be obtained, with $M$ being the total mass of the system. In particular, the density and the second-order moments are

$$
\rho(r)=\mu_{00}(r), \quad \rho \sigma_{r}^{2}(r)=\mu_{20}(r), \quad \rho \sigma_{T}^{2}(r)=\mu_{02}(r),
$$

and $\sigma_{T}^{2}(r)=2 \sigma_{\theta}^{2}(r)$. The density slope and the velocity anisotropy profile are defined as

$$
\gamma(r)=-\frac{d \ln \rho}{d \ln r}(r)
$$




$$
\beta(r)=1-\frac{\sigma_{\theta}^{2}(r)}{\sigma_{r}^{2}(r)} .
$$

Spherical dynamical models satisfy the Jeans equation

$$
\frac{d \rho \sigma_{r}^{2}}{d r}(r)+\frac{2 \beta(r)}{r} \rho \sigma_{r}^{2}(r)=\rho(r) \frac{d \psi}{d r}(r),
$$

which can be written as

$$
\sigma_{r}^{2}(r)(\gamma(r)-2 \beta(r)+\kappa(r))=v_{c}^{2}(r),
$$

with

$$
\kappa(r)=-\frac{d \ln \sigma_{r}^{2}}{d \ln r}(r), \quad v_{c}^{2}(r)=-r \frac{d \psi}{d r}(r) .
$$

Evidently, it follows that

$$
\gamma(r)-2 \beta(r)+\kappa(r) \geqslant 0, \quad \forall r .
$$

Ciotti \& Morganti (2010a, 2010b) showed that several systems (see the introduction) satisfy a stronger condition, the GDSAI

$$
\gamma(r)-2 \beta(r) \geqslant 0, \quad \forall r,
$$

and they pose the question whether this condition holds for all spherical systems. Naturally, the inequality is valid outside the radius $r_{2}$ where $\gamma\left(r_{2}\right)=2$. It is also valid at $r=0$, as was proven by An \& Evans (2006). In this paper, we will investigate the GDSAI for a particular class of systems, namely, those with a separable augmented density.

\section{THE AUGMENTED DENSITY CONCEPT}

A spherical dynamical system can also described by an augmented velocity moment (Dejonghe 1986; Paper I), which extends the moment to an explicit function $\tilde{\mu}_{2 n, 2 m}(\psi, r)$ of both the radius and the gravitational potential. An augmented moment is equivalent to the DF; the knowledge of one augmented moment determines the entire system. In particular, we will consider the augmented density $\tilde{\rho}(\psi, r)$, and its relationship with the DF is given by

$$
\tilde{\rho}(\psi, r)=2 \pi M \int_{0}^{\psi} d E \int_{0}^{2(\psi-E)} \frac{F\left(E, r v_{T}\right)}{\sqrt{2(\psi-E)-v_{T}^{2}}} d v_{T}^{2} .
$$

This integral equation can in principle be inverted to obtain the DF by using Laplace-Mellin transforms, although in practice the inversion is only numerically stable for sufficiently smooth systems. The strength of the augmented density framework lies in its direct connection to observable quantities like the velocity moments. For instance, the augmented velocity dispersion profiles are given by

$$
\begin{gathered}
\tilde{\sigma}_{r}^{2}(\psi, r)=\frac{1}{\tilde{\rho}(\psi, r)} \int_{0}^{\psi} \tilde{\rho}\left(\psi^{\prime}, r\right) d \psi^{\prime}, \\
\tilde{\sigma}_{T}^{2}(\psi, r)=\frac{2}{\tilde{\rho}(\psi, r)} \int_{0}^{\psi} D_{r^{2}}\left[r^{2} \tilde{\rho}\left(\psi^{\prime}, r\right)\right] d \psi^{\prime},
\end{gathered}
$$

where $D_{r^{2}}$ denotes the derivative with respect to $r^{2}$. The observed density and dispersions are then simply recovered from

$$
\rho(r)=\tilde{\rho}(\psi(r), r),
$$

$$
\begin{aligned}
& \sigma_{r}^{2}(r)=\tilde{\sigma}_{r}^{2}(\psi(r), r), \\
& \sigma_{T}^{2}(r)=\tilde{\sigma}_{T}^{2}(\psi(r), r),
\end{aligned}
$$

and the density slope is

$$
\gamma(r)=-\frac{r}{\rho} \frac{\partial \tilde{\rho}}{\partial r}(\psi(r), r)-\frac{r}{\rho} \frac{d \psi}{d r}(r) \frac{\partial \tilde{\rho}}{\partial \psi}(\psi(r), r) .
$$

As remarked in the Introduction, Ciotti \& Morganti have examined the GDSAI in several systems with a separable augmented density, i.e., systems of the form

$$
\tilde{\rho}(\psi, r)=f(\psi) g(r), \quad 0 \leqslant \psi \leqslant \psi_{0},
$$

with $\psi_{0}=\psi(0)$. For such models, the dispersion profiles read

$$
\begin{gathered}
\tilde{\sigma}_{r}^{2}(\psi)=\frac{1}{f(\psi)} \int_{0}^{\psi} f\left(\psi^{\prime}\right) d \psi^{\prime}, \\
\tilde{\sigma}_{T}^{2}(\psi, r)=\left(1+\frac{1}{2} \frac{d \ln g}{d \ln r}\right) \frac{2}{f(\psi)} \int_{0}^{\psi} f\left(\psi^{\prime}\right) d \psi^{\prime} .
\end{gathered}
$$

Note that the radial velocity dispersion is now only a function of $\psi$. The velocity anisotropy profile of these systems has the simple form

$$
\beta(r)=-\frac{1}{2} \frac{d \ln g}{d \ln r}(r) .
$$

As we demonstrated in Paper I and Paper II, this property provides a very elegant way to construct dynamical models with a given potential, density, and velocity anisotropy. Indeed, separable systems are completely determined by $\psi(r), \rho(r)$, and $\beta(r)$, since $g(r)$ is defined by Equation (23) and, by inverting $\psi(r)$, the function $f(\psi)$ follows from

$$
f(\psi)=\frac{\rho(r(\psi))}{g(r(\psi))} .
$$

However, one still needs to verify whether the corresponding DF is non-negative everywhere. Equation (19) now reduces to

$$
\gamma(r)=-\frac{d \ln g}{d \ln r}(r)-\frac{d \ln \psi}{d \ln r}(r) \frac{d \ln f}{d \ln \psi}(\psi(r)),
$$

so that we obtain

$$
\frac{d f}{d \psi}(\psi(r))=\frac{f(\psi(r))}{v_{c}^{2}(r)}(\gamma(r)-2 \beta(r)) .
$$

In other words, as remarked by Ciotti \& Morganti, the GDSAI

$$
\gamma(r) \geqslant 2 \beta(r), \quad \forall r \geqslant 0,
$$

is for separable systems equivalent to the statement

$$
\frac{d f}{d \psi} \geqslant 0, \quad \forall 0 \leqslant \psi \leqslant \psi_{0} .
$$

The question thus becomes whether this inequality is valid for all separable systems. In the following section, we will prove that this is indeed the case, if $\beta_{0} \leqslant 1 / 2$. 


\section{ANALYSIS OF THE GDSAI FOR SEPARABLE SYSTEMS}

Following the reasoning of An \& Evans (2006), we assume that any well-behaved DF can be written in the form

$$
F(E, L)=L^{-2 \beta_{0}}\left(F_{0}(E)+F_{1}(E, L)\right)
$$

with

$$
F_{1}(E, 0) \equiv 0, \quad \forall 0 \leqslant E \leqslant \psi_{0} .
$$

The function $L^{-2 \beta_{0}} F_{0}(E)$ in this Ansatz can be understood as the leading term of a Laurent series expansion in $L$ at $L=0$. Toward the center $r \rightarrow 0$, the DF is dominated by this term, which has the form of a system with constant anisotropy. Consequently, the central anisotropy of the entire model indeed corresponds with $\beta_{0}$. Since the DF has to be non-negative everywhere, it follows immediately that $F_{0}(E) \geqslant 0 \forall E$ is a necessary condition to obtain a physically meaningful DF.

If we consider separable systems, the corresponding augmented density then has the form

$$
\tilde{\rho}(\psi, r)=f(\psi) r^{-2 \beta_{0}}\left(1+g_{1}(r)\right), \quad \text { with } g_{1}(0)=0
$$

Using $u^{2}=\frac{v_{T}^{2}}{2(\psi-E)}$, the relation between the augmented density and the DF (13) can be written as

$$
\begin{aligned}
\tilde{\rho}(\psi, r)= & 2 \pi 2^{1 / 2-\beta_{0}} r^{-2 \beta_{0}} M \int_{0}^{1} \frac{u^{-2 \beta_{0}}}{\sqrt{1-u^{2}}} d u^{2} \\
& \times \int_{0}^{\psi}(\psi-E)^{1 / 2-\beta_{0}}\left(F_{0}(E)\right. \\
& \left.+F_{1}(E, r u \sqrt{2(\psi-E)})\right) d E .
\end{aligned}
$$

In separable systems, it follows that

$$
f(\psi)=\frac{\tilde{\rho}(\psi, r)}{g(r)} .
$$

Since the left-hand side of this equation is independent of the radius $r$, the right-hand side does not depend on $r$ either. The equality is therefore valid for all values $r$; in particular, we can take the limit of $r$ toward the center,

$$
f(\psi)=\lim _{r \rightarrow 0} \frac{\tilde{\rho}(\psi, r)}{g(r)}=\lim _{r \rightarrow 0} r^{2 \beta_{0}} \tilde{\rho}(\psi, r) .
$$

This property is the key element to prove the GDSAI when $\beta_{0} \leqslant 1 / 2$ : using Equations (30) and (34), it follows from Equation (32) that

$f(\psi)=(2 \pi)^{3 / 2} 2^{-\beta_{0}} M \frac{\Gamma\left(1-\beta_{0}\right)}{\Gamma\left(3 / 2-\beta_{0}\right)} \int_{0}^{\psi}(\psi-E)^{1 / 2-\beta_{0}} F_{0}(E) d E$.

Remarkably, the function $f(\psi)$ thus only depends on $F_{0}(E)$ and $\beta_{0}$. In other words, for separable systems the function $F_{1}(E, L)$ has no influence on the GDSAI. Concrete examples of this behavior are furnished in the systems considered by Ciotti \& Morganti. For instance, the equivalent function $B\left(\psi_{\mathrm{T}}\right)$ in Ciotti $\&$ Morganti (2010b) for generalized Cuddeford systems does not depend on the anisotropy radius $r_{\mathrm{a}}$ (see their Equation (13)).

The value of $\beta_{0}$ splits our further analysis into three cases: $\beta_{0}<1 / 2, \beta_{0}=1 / 2$, and $\beta_{0}>1 / 2$.

\subsection{Prooffor $\beta_{0}<1 / 2$}

If $\beta_{0}<1 / 2$, the derivative of $f(\psi)$ becomes

$$
\begin{aligned}
\frac{d f}{d \psi}(\psi)= & (2 \pi)^{3 / 2} 2^{-\beta_{0}} M \frac{\Gamma\left(1-\beta_{0}\right)}{\Gamma\left(3 / 2-\beta_{0}\right)}\left[\lim _{E \rightarrow \psi}(\psi-E)^{1 / 2-\beta_{0}} F_{0}(E)\right. \\
& \left.+\left(\frac{1}{2}-\beta_{0}\right) \int_{0}^{\psi}(\psi-E)^{-1 / 2-\beta_{0}} F_{0}(E) d E\right] .
\end{aligned}
$$

Let us examine the first term inside the brackets: if

$$
\lim _{E \rightarrow \psi}(\psi-E)^{1 / 2-\beta_{0}} F_{0}(E)>0,
$$

then

$$
\lim _{E \rightarrow \psi}(\psi-E)^{-1 / 2-\beta_{0}} F_{0}(E) \sim \lim _{E \rightarrow \psi}(\psi-E)^{-a} \quad \text { with } a \geqslant 1,
$$

so that

$$
\int_{0}^{\psi}(\psi-E)^{-1 / 2-\beta_{0}} F_{0}(E) d E=+\infty .
$$

In other words, if the limit is nonzero, then the integral in the second term becomes infinite. The limit can therefore be omitted, so that the equation is simplified to

$$
\begin{aligned}
\frac{d f}{d \psi}(\psi)= & (2 \pi)^{3 / 2} 2^{-\beta_{0}} M \frac{\Gamma\left(1-\beta_{0}\right)}{\Gamma\left(1 / 2-\beta_{0}\right)} \\
& \times \int_{0}^{\psi} \frac{F_{0}(E)}{(\psi-E)^{1 / 2+\beta_{0}}} d E \geqslant 0,
\end{aligned}
$$

and recalling Equation (26), the GDSAI is proven. The above relation can be generalized further: if $n=\left\lfloor 3 / 2-\beta_{0}\right\rfloor$ and $\alpha=3 / 2-\beta_{0}-n$ are the integer floor and fractional part of $3 / 2-\beta_{0}$, then

$$
\begin{aligned}
\frac{d^{k} f}{d \psi^{k}}(\psi)= & (2 \pi)^{3 / 2} 2^{-\beta_{0}} M \frac{\Gamma\left(1-\beta_{0}\right)}{\Gamma\left(3 / 2-\beta_{0}-k\right)} \\
& \times \int_{0}^{\psi}(\psi-E)^{1 / 2-\beta_{0}-k} F_{0}(E) \geqslant 0, \quad 0 \leqslant k \leqslant n,
\end{aligned}
$$

so the inequalities

$$
\frac{d^{k} f}{d \psi^{k}}(\psi) \geqslant 0, \quad \forall 0 \leqslant \psi \leqslant \psi_{0}, \quad 0 \leqslant k \leqslant n,
$$

are necessary conditions to obtain a separable system with a non-negative DF. This extends the results obtained by Ciotti \& Morganti (2010a) for multi-component Cuddeford models.

$$
\text { 4.2. Prooffor } \beta_{0}=1 / 2
$$

When $\beta_{0}=1 / 2$, Equation (35) reduces to

$$
f(\psi)=2 \pi^{2} M \int_{0}^{\psi} F_{0}(E) d E .
$$

The derivative is then simply

$$
\frac{d f}{d \psi}(\psi)=2 \pi^{2} M F_{0}(\psi) \geqslant 0,
$$

so evidently, the GDSAI is again a necessary condition for a physical dynamical model. 


\subsection{Counterexamples for $\beta_{0}>1 / 2$}

The proof is not applicable to systems with $\beta_{0}>1 / 2$. Indeed, the derivative has the same form as Equation (45), but now the two terms inside the brackets are, respectively, $+\infty$ and $-\infty$ when $F_{0}(E)>0$, so their sum is undetermined. However, we can rewrite Equation (35) using integration by parts as

$$
\begin{aligned}
f(\psi)= & (2 \pi)^{3 / 2} 2^{-\beta_{0}} M \frac{\Gamma\left(1-\beta_{0}\right)}{\Gamma\left(5 / 2-\beta_{0}\right)}\left[\psi^{3 / 2-\beta_{0}} F_{0}(0)\right. \\
& \left.+\int_{0}^{\psi}(\psi-E)^{3 / 2-\beta_{0}} F_{0}^{\prime}(E) d E\right]
\end{aligned}
$$

where $F_{0}^{\prime}(E)$ denotes the derivative of $F_{0}(E)$. After differentiation, we then obtain

$$
\begin{aligned}
\frac{d f}{d \psi}(\psi)= & (2 \pi)^{3 / 2} 2^{-\beta_{0}} M \frac{\Gamma\left(1-\beta_{0}\right)}{\Gamma\left(3 / 2-\beta_{0}\right)}\left[\psi^{1 / 2-\beta_{0}} F_{0}(0)\right. \\
& \left.+\int_{0}^{\psi}(\psi-E)^{1 / 2-\beta_{0}} F_{0}^{\prime}(E) d E\right] .
\end{aligned}
$$

Thus, separable systems with a monotonically increasing $F_{0}(E)$ (i.e., $F_{0}^{\prime}(E) \geqslant 0 \quad \forall E$ ) satisfy the GDSAI. Again, this is an extension of the results for generalized Cuddeford systems found by Ciotti \& Morganti (2010b).

Yet, the GDSAI is no longer a necessary condition for a physical model, which raises the question whether systems can be found for which the global inequality does not hold. To this aim, we consider the potential-density pair

$$
\begin{gathered}
\psi(r)=\frac{G M_{\mathrm{tot}}}{(1+\sqrt{r})^{2}}, \\
\rho(r)=\frac{3 M}{8 \pi} \frac{1}{r^{3 / 2}(1+\sqrt{r})^{4}},
\end{gathered}
$$

with corresponding density slope

$$
\gamma(r)=\frac{3 / 2+7 / 2 \sqrt{r}}{1+\sqrt{r}},
$$

which is part of the family of Veltmann models or $\alpha$-models (Veltmann 1979; Zhao 1996), and was discussed by Moore et al. (1998). If $M_{\text {tot }}=M$, then the system is also self-consistent. For this pair, we construct physical DFs that generate four-parameter anisotropy profiles of the form

$$
\beta(r)=\frac{\beta_{0}+\beta_{\infty}\left(r / r_{\mathrm{a}}\right)^{2 \delta}}{1+\left(r / r_{\mathrm{a}}\right)^{2 \delta}},
$$

with $0<\delta \leqslant 1$, so that

$$
\tilde{\rho}(\psi, r)=f(\psi)\left(\frac{r}{r_{\mathrm{a}}}\right)^{-2 \beta_{0}}\left(1+\frac{r^{2 \delta}}{r_{\mathrm{a}}^{2 \delta}}\right)^{\beta_{\delta}}
$$

with

$$
\beta_{\delta}=\frac{\beta_{0}-\beta_{\infty}}{\delta} .
$$

Again, our systems have separable augmented densities. For every anisotropy profile, the function $f(\psi)$ follows from $\rho(r)=\tilde{\rho}(\psi(r), r)$, and the DF can be found by inverting Equation (13). Instead of performing these calculations directly, we adopt the technique used in Paper II; we first generate a family of components of the form

$$
\tilde{\rho}_{i}(\psi, r)=\rho_{0 i}\left(\frac{\psi}{\psi_{0}}\right)^{p_{i}}\left(1-\frac{\psi^{s_{i}}}{\psi_{0}^{s_{i}}}\right)^{q_{i}}\left(\frac{r}{r_{\mathrm{a}}}\right)^{-2 \beta_{0}}\left(1+\frac{r^{2 \delta}}{r_{\mathrm{a}}^{2 \delta}}\right)^{\beta_{\delta}}
$$

where $p_{i}, q_{i}$, and $s_{i}$ are three parameters, and $\rho_{0 i}$ are normalization constants. The corresponding DFs are (see Paper I; Paper II)

$$
\begin{aligned}
& F_{i}(E, L)=\frac{\rho_{0 i}}{M\left(2 \pi \psi_{0}\right)^{3 / 2}} \sum_{j=0}^{\infty}(-1)^{j}\left(\begin{array}{c}
q_{i} \\
j
\end{array}\right)\left(\frac{E}{\psi_{0}}\right)^{p_{i}+j s_{i}-3 / 2} \\
& \times \sum_{k=0}^{\infty}\left(\begin{array}{c}
\beta_{\delta} \\
k
\end{array}\right) \frac{\Gamma\left(1+p_{i}+j s_{i}\right)}{\Gamma\left(1-\beta_{0}+k \delta\right) \Gamma\left(p_{i}+j s_{i}-\frac{1}{2}+\beta_{0}-k \delta\right)} \\
& \quad \times\left(\frac{L^{2}}{2 r_{\mathrm{a}}^{2} E}\right)^{-\beta_{0}+k \delta}
\end{aligned}
$$

for $L^{2}<2 r_{\mathrm{a}}^{2} E$, and

$$
\begin{aligned}
& F_{i}(E, L)=\frac{\rho_{0 i}}{M\left(2 \pi \psi_{0}\right)^{3 / 2}} \sum_{j=0}^{\infty}(-1)^{j}\left(\begin{array}{c}
q_{i} \\
j
\end{array}\right)\left(\frac{E}{\psi_{0}}\right)^{p_{i}+j s_{i}-3 / 2} \\
& \quad \times \sum_{k=0}^{\infty}\left(\begin{array}{c}
\beta_{\delta} \\
k
\end{array}\right) \frac{\Gamma\left(1+p_{i}+j s_{i}\right)}{\Gamma\left(1-\beta_{\infty}-k \delta\right) \Gamma\left(p_{i}+j s_{i}-\frac{1}{2}+\beta_{\infty}+k \delta\right)} \\
& \quad \times\left(\frac{L^{2}}{2 r_{\mathrm{a}}^{2} E}\right)^{-\beta_{\infty}-k \delta}
\end{aligned}
$$

for $L^{2}>2 r_{\mathrm{a}}^{2} E$. With different values of the parameters, a library of base functions is thus created, from which a linear combination is built that fits the given density $\rho(r)$ at various radii. This is achieved by minimizing the quantity

$$
\chi_{N}^{2}=\frac{1}{N_{\text {data }}} \sum_{m=1}^{N_{\text {data }}} \frac{1}{\rho\left(r_{m}\right)}\left(\rho\left(r_{m}\right)-\sum_{i=1}^{N} a_{i} \rho_{i}\left(r_{m}\right)\right)^{2},
$$

using a quadratic programming algorithm (Dejonghe 1989). The details of this procedure can be found in Paper II. In particular, we created models with $N=12$ components, fitting 25 density data points extracted from Equation (48).

With this technique, we obtain several dynamical models with non-negative DFs that violate the GDSAI; three of them are shown in Figure 1. All three share the anisotropy parameters $\beta_{0}=0.75, \beta_{\infty}=1$, and $r_{\mathrm{a}}=0.02$ but have different values for $\delta: 0.3,0.6$, and 1.0 , respectively; note that the latter is a Cuddeford-type model. For the model with $\delta=0.3$, we find that $\gamma(r)<2 \beta(r)$ for radii in the interval [0,0.021], with a minimum around $r=0.0057$ (note that the center is a local maximum, for which $\gamma_{0}=2 \beta_{0}$ ). In the model with $\delta=0.6$, the $\gamma-\beta$ relation reaches a local maximum around $r=0.0028$, and the GDSAI does not hold in the interval [0.019, 0.061], with a minimum around $r=0.036$. Finally the largest $\gamma-\beta$ fluctuations occur in the Cuddeford model $(\delta=1)$, with a local maximum around $r=0.0054$, and a GDSAI violation within $[0.019,0.100]$, with a minimum for $r=0.044$.

Evidently, we require rather extreme parameter values to obtain these (modest) violations, while maintaining non-negative DFs. The central anisotropy $\beta_{0}$ has to be high, and the profile $\beta(r)$ has to increase very rapidly. It is therefore safe to assume 

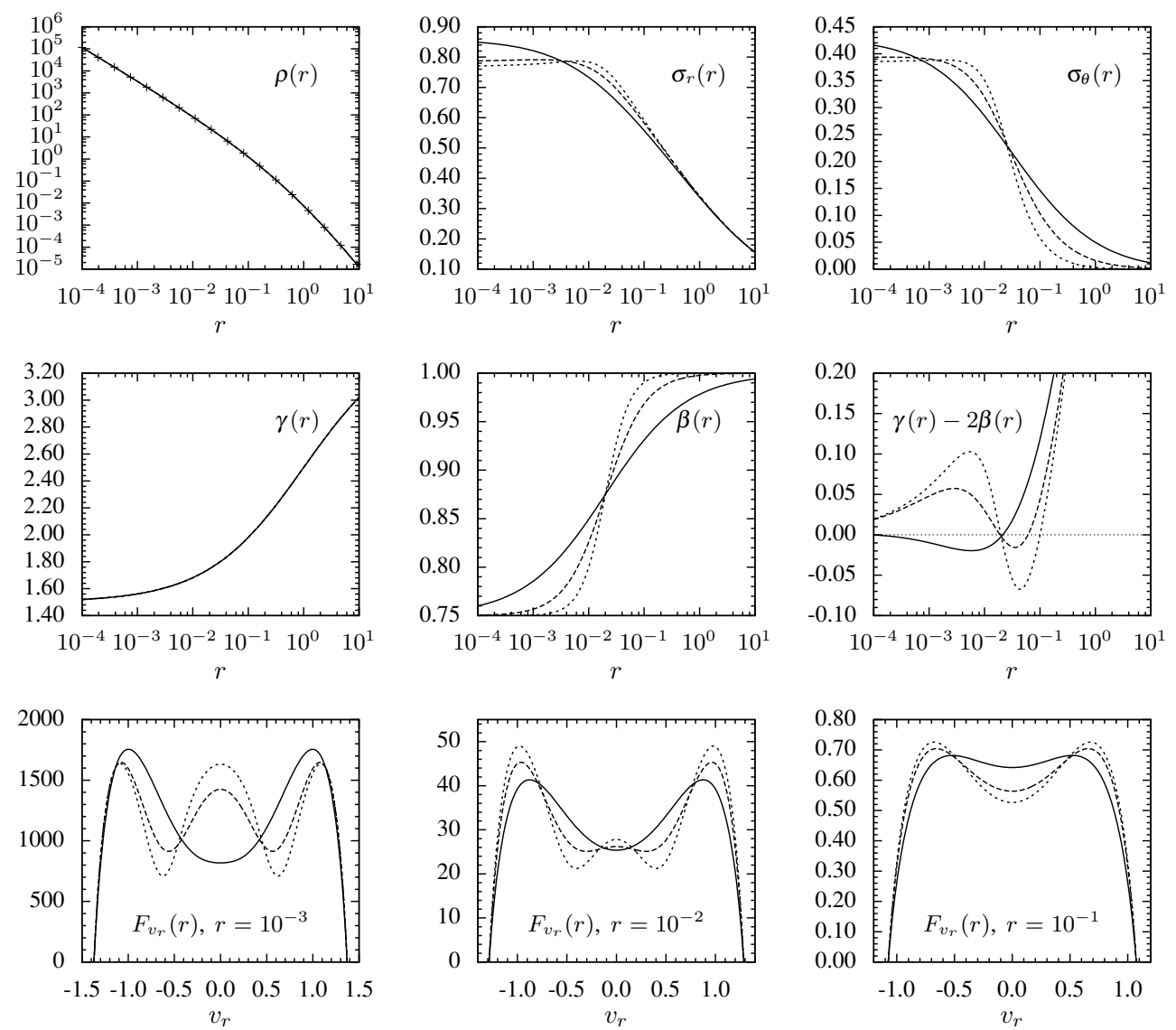

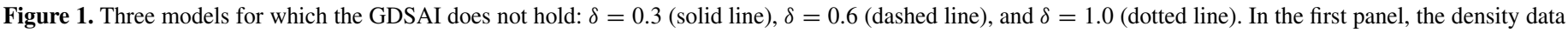
points are also displayed.

that the self-consistent variants of these models are dynamically unstable. This can be seen from the standard criterion for radial-orbit instability: $2 T_{r} / T_{T}=2\left\langle v_{r}\right\rangle /\left\langle v_{T}\right\rangle=5.45,8.26$, and 10.42 for the three models, which is much higher than the $\simeq 2$ threshold for similar models (see Merritt 1999 for an overview). Further evidence of dynamical instability is given by the radial velocity distributions

$$
F_{v_{r}}(r)=2 \pi M \int_{0}^{\sqrt{2 \psi(r)-v_{r}^{2}}} F(E, L) v_{T} d v_{T} .
$$

As shown in the bottom row of Figure 1, these profiles have two or three peaks at small radii. These are indications of Hénon instabilities (see Merritt 1999; Barnes et al. 1986). In theory, if the systems are instead not self-consistent but embedded in a massive dark matter halo, they might withstand these instabilities; however, one can safely argue that such equilibrium systems are too extreme to arise in structure formation.

\subsection{The Inverse Relation}

Finally, we remark that the function $F_{0}(E)$ can be derived from $f(\psi)$ by means of an Abel-related inversion (Cuddeford 1991; An \& Evans 2006), which holds for all values of $\beta_{0}<1$,

$$
\begin{aligned}
F_{0}(E)= & \frac{2^{\beta_{0}}}{(2 \pi)^{3 / 2} M \Gamma(1-\alpha) \Gamma\left(1-\beta_{0}\right)} \\
& \times\left(\int_{0}^{E} \frac{d^{n+1} f}{d \psi^{n+1}} \frac{d \psi}{(E-\psi)^{\alpha}}+\frac{1}{E^{\alpha}} \frac{d^{n} f}{d \psi^{n}}(0)\right),
\end{aligned}
$$

where again $n=\left\lfloor 3 / 2-\beta_{0}\right\rfloor$ and $\alpha=3 / 2-\beta_{0}-n$ are the integer floor and fractional part of $3 / 2-\beta_{0}$. Thus, the additional condition

$$
\frac{d^{n+1} f}{d \psi^{n+1}}(\psi) \geqslant 0, \quad \forall 0 \leqslant \psi \leqslant \psi_{0},
$$

is sufficient to obtain a non-negative $F_{0}(E)$. As Ciotti \& Morganti (2010a) showed, this also implies that the entire DF $F(E, L)$ is non-negative in the case of generalized Cuddeford systems. However, it is not a priori clear whether this property is true for all separable systems, since the behavior of $F_{1}(E, L)$ might still lead to negative values of the DF. Further study is therefore needed to determine if Equation (59) is a sufficient condition for the existence of a physical separable model.

\section{DISCUSSION}

In the previous section, we presented a full analysis of the GDSAI for spherical dynamical systems with a separable augmented density. As our proof shows, the GDSAI holds if the central velocity anisotropy $\beta_{0} \leqslant 1 / 2$. We further demonstrated that for systems with $\beta_{0}>1 / 2$, the GDSAI can be broken, as shown by three counterexamples, although these systems are not physically realistic.

Equations (40) and (44), combined with Equation (26), also reveal a remarkable property of separable systems: the GDSAI is purely determined by $\beta_{0}$ and $F_{0}(E)$. The function $F_{0}(E)$ can be interpreted in various ways: it can be thought of as the phase-space distribution of particles at purely radial orbits, as 
the phase-space distribution of particles at the center, or as the energy distribution of the constant-anisotropy component of the DF. As a surprising consequence, if a separable system has a given potential $\psi(r)$ and density $\rho(r)$, then knowledge of $F_{0}(E)$ alone is sufficient to construct the complete DF of the system. Indeed, we showed that $F_{0}(E)$ is equivalent with $f(\psi)$, and in combination with $\rho(r)$, the function $g(r)=\rho(r) / f(\psi(r))$ can also be derived, determining the augmented density $f(\psi) g(r)$ and thus $F(E, L)$.

The next logical step will be to investigate the GDSAI for general, non-separable spherical models. One possible approach would be to consider a spherical system as a linear combination of separable systems. In fact, an analytic $\tilde{\rho}(\psi, r)$ or $F(E, L)$ can be written as a double sum of power-law functions, by means of a two-dimensional Laurent series expansion. An alternative approach would be to ask the following question: given a spherical dynamical system with a given $\psi(r)$ and a DF that generates $\rho(r)$ and $\beta(r)$, does there always exist a separable model with a non-negative DF that generates the same density and anisotropy? As we mentioned in Section 3, the function $g(r)$ follows directly from $\beta(r)$, and in turn this determines $f(\psi)$ from $g(r)$ and $\rho(r)$. However, there is no a priori reason that the corresponding DF is also non-negative. If it is, the same GDSAI analysis applies as presented in this paper. We currently do not know of any $(\rho(r), \beta(r))$ pair that can only be generated by non-separable models, but more study is required to resolve these questions. We think that our analysis of separable systems can be a useful stepping stone for further investigations of the GDSAI for spherical dynamical systems.

The authors thank the referee Luca Ciotti for the generous comments and helpful suggestions that improved our paper.

\section{REFERENCES}

An, J. H., \& Evans, N. W. 2006, ApJ, 642, 752

Baes, M., \& Dejonghe, H. 2002, A\&A, 393, 485

Baes, M., \& van Hese, E. 2007, A\&A, 471, 419

Barnes, J., Hut, P., \& Goodman, J. 1986, ApJ, 300, 112

Buyle, P., Hunter, C., \& Dejonghe, H. 2007, MNRAS, 375, 773

Ciotti, L., \& Morganti, L. 2010a, MNRAS, 401, 1091

Ciotti, L., \& Morganti, L. 2010b, MNRAS, 408, 1070

Cuddeford, P. 1991, MNRAS, 253, 414

Cuddeford, P., \& Louis, P. 1995, MNRAS, 275, 1017

Dejonghe, H. 1986, Phys. Rep., 133, 217

Dejonghe, H. 1987, MNRAS, 224, 13

Dejonghe, H. 1989, ApJ, 343, 113

Evans, N. W., \& An, J. 2005, MNRAS, 360, 492

Merritt, D. 1985, AJ, 90, 1027

Merritt, D. 1999, PASP, 111, 129

Moore, B., Governato, F., Quinn, T., Stadel, J., \& Lake, G. 1998, ApJ, 499, L5

Osipkov, L. P. 1979, Pis'ma Astron. Zh., 5, 77

Van Hese, E., Baes, M., \& Dejonghe, H. 2009, ApJ, 690, 1280

Veltmann, U. I. K. 1979, Astron. Zh., 56, 976

Zhao, H. 1996, MNRAS, 278, 488 